OPEN ACCESS

Check for updates

\section{Suicide and self-harm in adult survivors of critical illness: population based cohort study}

\author{
Shannon M Fernando, ${ }^{1,2}$ Danial Qureshi, ${ }^{3,4,5,6}$ Manish M Sood, ${ }^{3,4,5,7}$ Michael Pugliese, 3,4 \\ Robert Talarico,, Daniel T Myran, ${ }^{3,4,8}$ Margaret S Herridge, ${ }^{9,10,11}$ Dale M Needham, ${ }^{12,13}$ \\ Bram Rochwerg, ${ }^{14,15}$ Deborah J Cook, ${ }^{14,15}$ Hannah Wunsch, ${ }^{3,9,11,16}$ Robert A Fowler, ${ }^{3,9,11,16}$ \\ Damon C Scales, ${ }^{3,9,11,16,17}$ O Joseph Bienvenu, ${ }^{18}$ Kathryn M Rowan, ${ }^{19}$ Magdalena Kisilewicz, ${ }^{20}$ \\ Laura H Thompson, ${ }^{4}$ Peter Tanuseputro, ${ }^{3,4,5,6,21}$ Kwadwo Kyeremanteng ${ }^{1,4,21,22}$
}

For numbered affiliations see end of the article.

Correspondence to:

SM Fernando, Department of Critical Care, The Ottawa Hospital, General Campus, 501 Smyth Rd, Ottawa, ON, Canada K1H 8M2 sfernando@qmed.ca

(or@shanfernands on Twitter: ORCID 0000-0003-4549-4289) Additional material is published online only. To view please visit the journal online.

Cite this as: $B M J$ 2021;373:n973 http://dx.doi.org/10.1136/bmj.n973

Accepted: 7 April 2021

\section{ABSTRACT}

OBJECTIVE

To analyse the association between survival from critical illness and suicide or self-harm after hospital discharge.

\section{DESIGN}

Population based cohort study using linked and validated provincial databases.

SETTING

Ontario, Canada between January 2009 and December 2017 (inclusive).

\section{PARTICIPANTS}

Consecutive adult intensive care unit (ICU) survivors ( $\geq 18$ years) were included. Linked administrative databases were used to compare ICU hospital survivors with hospital survivors who never required ICU admission (non-ICU hospital survivors). Patients were categorised based on their index hospital admission (ICU or non-ICU) during the study period.

MAIN OUTCOME MEASURES

The primary outcome was the composite of death by suicide (as noted in provincial death records) and deliberate self-harm events after discharge. Each outcome was also assessed independently. Incidence of suicide was evaluated while accounting for competing risk of death from other causes. Analyses were conducted by using overlap propensity score models. weighted, cause specific Cox proportional hazard

\section{WHAT IS ALREADY KNOWN ON THIS TOPIC}

Survival after critical illness is associated with important sequelae, including muscle weakness, reduced exercise capacity, fatigue, cognitive impairment, pain, and financial hardship

Growing evidence shows that intensive care unit (ICU) survivors have higher rates of psychiatric morbidity, but whether that translates into an increased risk of suicide and self-harm is unknown

\section{WHAT THIS STUDY ADDS}

In this population based study of 423060 consecutive ICU survivors, ICU admission was associated with higher risk of suicide or self-harm after hospital discharge compared with non-ICU hospital admission

Risk of suicide and self-harm was associated with younger age, previous mental health diagnoses, and receipt of life support interventions

ICU admission could be a risk factor for downstream suicide and self-harm, and early intervention among particular patient populations might be helpful in attenuating this risk

\section{RESULTS}

423060 consecutive ICU survivors (mean age 61.7 years, $39 \%$ women) were identified. During the study period, the crude incidence (per 100000 person years) of suicide, self-harm, and the composite of suicide or self-harm among ICU survivors was 41.4 , 327.9 , and 361.0, respectively, compared with 16.8 , 177.3, and 191.6 in non-ICU hospital survivors. Analysis using weighted models showed that ICU survivors ( $v$ non-ICU hospital survivors) had a higher risk of suicide (adjusted hazards ratio 1.22, 95\% confidence interval 1.11 to 1.33) and self-harm (1.15, 1.12 to 1.19). Among ICU survivors, several factors were associated with suicide or self-harm: previous post-traumatic stress disorder $(1.87,1.64$ to 2.13$)$, invasive mechanical ventilation $(1.45,1.38$ to 1.54$)$, and renal replacement therapy $(1.35,1.17$ to 1.56$)$.

CONCLUSIONS

Survivors of critical illness have increased risk of suicide and self-harm, and these outcomes were associated with pre-existing psychiatric illness and receipt of invasive life support. Knowledge of these prognostic factors might allow for earlier intervention to potentially reduce this important public health problem.

\section{Introduction}

In North America, millions of critically ill patients are admitted to the intensive care unit (ICU) annually. With expanding critical care capacity and an ageing population, the number of ICU patients is expected to increase globally. ${ }^{1}$ Historically, critically ill patients have experienced high rates of mortality. However, substantial advances in the field of critical care have led to improved survival over the past two decades. ${ }^{23}$ With an increasing number of ICU survivors, a growing need exists for understanding and improving the long lasting morbidity that these patients might experience. ${ }^{45}$ Sequelae experienced by survivors of critical illness include muscle weakness, reduced exercise capacity, fatigue, cognitive impairment, pain, poor quality of life, and financial hardship..$^{5-11}$ Recent evidence has also shown an association between critical illness and psychiatric morbidity. ${ }^{12}$ The combination of acute illness severity and resultant morbidity in ICU survivors (frequently called postintensive care syndrome) combine to make this group highly vulnerable to mental suffering. More specifically, ICU survivorship has been shown to be associated depression or anxiety $(5.69,5.38$ to 6.02$)$, previous 
with increased incidences of depression, anxiety, post-traumatic stress disorder (PTSD), substance use disorders, and psychosis. ${ }^{11-15}$

This increased risk of psychiatric morbidity in ICU survivors might lead to increased suicide and selfharm behaviours. Suicide is the 11th leading cause of death worldwide (second leading cause among adults younger than 35), and the incidence of suicide is increasing. ${ }^{16}$ Identification of high risk populations can help to mitigate the risk of harm. ${ }^{17}$ However, the incidence of suicide and self-harm among ICU survivors, associated risk factors, and how this incidence compares with hospital survivors who did not require ICU admission is unknown. Therefore, we used population level administrative data from the province of Ontario, Canada to investigate the incidence and risk factors associated with suicide and self-harm behaviours among adult survivors of critical illness, and compared the associated risk with hospital survivors who did not require ICU admission.

\section{Methods}

Studies conducted at ICES using administrative data fall under section 45 of the Personal Health Information Protection Act of Ontario, and do not require research ethics board approval.

\section{Data sources and setting}

We conducted a population level cohort study using health administrative databases from the province of Ontario in Canada (population 14.6 million). Within Ontario's single payer healthcare system, all publicly funded healthcare services, physician, hospital, and demographic information for residents are recorded in these databases. These datasets were linked using unique encoded identifiers, and analysed at ICES, an independent, non-profit research institute whose legal status under Ontario's health information privacy law allows it to collect and analyse healthcare and demographic data, without consent, for health system evaluation and improvement. ${ }^{18}$ ICES is funded by an annual grant from the Ontario Ministry of Health and Long-term Care.

We linked nine databases at ICES, at the individual patient level, from 1 January 2009 to 31 December 2017 (supplemental table 1). Data on cause of death (including suicide) was obtained from the Vital Statistics Database (Office of the Registrar General). Data contained in ICES is full and complete, with the exception of emigration from Ontario, which represents approximately $0.5 \%$ of patients per year. ${ }^{19}$

\section{Patients}

We included all adult patients ( $\geq 18$ years) with an index hospital admission in Ontario between 1 January 2009 and 31 December 2017, and who survived to hospital discharge (survivors). For patients with multiple hospital admissions, we only considered the first admission during this study period. We identified adult patients admitted to an ICU setting during the study period (ICU survivors) by using previously validated algorithms from the Canadian Institute for Health Information Discharge Abstract Database. ${ }^{1420} 21$ We compared these patients with those admitted to a hospital setting during the study period who never required ICU (non-ICU hospital survivors).

At the time of the index hospital admission, we identified important patient characteristics, including age, sex, Charlson comorbidity index (CCI), ${ }^{22}$ date of admission, and the number of hospital admissions in the previous year. CCI was categorised on the basis of prevalence rates seen in previous studies ${ }^{23}$ (thresholds of $\leq 2,92.8 \%$ of patients admitted to hospital; 3-4, $4.6 \%$ of patients admitted to hospital; and $\geq 5,2.6 \%$ of patients admitted to hospital). We calculated duration of ICU and hospital length of stay from admission and discharge dates. We obtained income group (categorised into fifths) and rurality through postal code conversion files based on Statistics Canada census data. Given the strong association between preexisting mental health diagnoses and risk of suicide, ${ }^{17}$ we captured history of pre-existing mental health diagnoses through the use of ICD-10 (international classification of diseases, version 10) codes and whether patients had any outpatient mental health visits with a primary care provider or psychiatrist in the previous year (supplemental table 2). Finally, we recorded life support interventions received during hospital admission: invasive mechanical ventilation delivered through an endotracheal or tracheostomy tube; non-invasive mechanical ventilation delivered through a face mask; and renal replacement therapy (supplemental table 2).

\section{Outcomes}

The primary outcome was incidence of the composite of death by suicide or hospital visit for deliberate self-harm at any point after hospital discharge and before the end of the study period, as defined by previously validated ICD-10 codes (supplemental table 2). ${ }^{24}$ We also separately evaluated rates of death by suicide and hospital visit for deliberate self-harm. Self-harm behaviours were evaluated because they are strongly associated with subsequent death by suicide (96\% concordance with coroners' reports in Ontario). ${ }^{25}$ The ICD codes for death by suicide have strong concordance with Ontario coroner reported suicide, ${ }^{26}$ and have been used in previous studies. ${ }^{27}$ Self-harm behaviour (eg, deliberate drug overdose or self-inflicted traumatic injury) were identified by using the ICES Mental Health and Addictions Scorecard and Evaluation Framework, ${ }^{28}$ as previously reported. ${ }^{27} 29$

\section{Statistical analyses}

We conducted all statistical analyses with SAS Enterprise Guide 7.1 (SAS Institute, Cary, NC). We present data as mean values with standard deviations, or medians with interquartile ranges. We used cumulative incidence function curves to describe suicide and self-harm events in the follow-up, while adjusting for competing risk of death due to other causes. We used Gray's test to assess for equality. Similarly, all 
incidence rates account for competing risk of death due to other causes.

To identify the association between ICU admission and suicide or self-harm, we followed recommendations for causal inference in critical care, ${ }^{30}$ and compared ICU survivors with non-ICU hospital survivors using overlap propensity score weighting. Overlap weights is a technique that assigns less weight to those with outlier propensity scores and more weight to those with propensity scores close to 0.5 . This technique has shown improved covariate balance and overlap of probabilities in creating propensity scores compared with other approaches. ${ }^{31}$ Furthermore, this method does not exclude any potential study participants and is not affected by extreme outliers over dominating the analysis, which can occur with traditional propensity score matching or inverse probability treatment weighting. We calculated weights using relevant variables determined a priori (based on existing literature $^{12-17}$ and hypotheses ${ }^{12} 17$ about potential for confounding, as recommended ${ }^{30}$ ), including age, sex, year of index admission, number of hospital admissions in the past year, number of outpatient psychiatry visits in the past year, number of outpatient physician mental health visits in the past year, number of total outpatient physician visits in the past year, income fifth, rural residency, CCI, hospital length of stay, pre-existing mental health diagnoses, previous history of self-harm, and discharge disposition location. Supplemental table 1 includes an acyclic graph. We compared cohorts before and after overlap weights for balance by using weighted standardised differences. We fit an overlap weighted cause specific Cox proportional hazards model to examine ICU exposure versus non-ICU hospital admission with the study outcomes, while accounting for non-suicide related mortality as a competing risk. To account for unmeasured confounders, we computed an E value. ${ }^{32}$ A large $E$ value implies that considerable unmeasured confounding would be needed to explain away the effect estimate. To further evaluate for nonproportional hazards, we repeated our analyses using time from discharge as an interaction term.

To identify prognostic factors associated with suicide, deliberate self-harm, and the composite of these two outcomes among ICU survivors, we generated separate cause specific Cox proportional hazards regression models using the ICU survivors cohort. In model creation, we followed the PROGRESS (prognosis research strategy) guidelines, ${ }^{33}$ and recommendations for development of prognostic models in critical care. ${ }^{35}$ These guidelines recommend a clinically hypothesis driven approach for a priori selection of all model variables, as opposed to bivariate association testing methods. As recommended, 33-35 variables were selected based on theory or known evidence. ${ }^{12-17}$ The variables included in our model were age, sex, year of index admission, number of hospital admissions in the past year, income fifth, rural residency, CCI, hospital length of stay, ICU length of stay, invasive mechanical ventilation during hospital admission, renal replacement therapy during hospital admission, pre-existing mental health diagnoses, and discharge disposition. A total of 1805 patients $(0.4 \%$ of the cohort) had missing data related to income fifth and rural residency, and were subsequently excluded from the model. Finally, we compared crude incidence of suicide, self-harm, and the composite of these two outcomes in subgroups stratified by age, receipt of invasive therapies (invasive mechanical ventilation or renal replacement therapy), and pre-existing mental health diagnoses.

\section{Patient and public involvement}

This was a retrospective evaluation of existing health data, and great care was taken to preserve confidentiality. All investigators signed a nondisclosure agreement with regard to data preservation and confidentiality. Therefore, it was difficult to involve patients or members of the public in study design, interpretation of the data, or manuscript creation. However, we do acknowledge the potential importance of this study's findings for patients who might ultimately require critical care, and therefore will seek to disseminate our work through patient networks at our local institutions, and national organisations including the Canadian Critical Care Society, the Canadian Critical Care Trials Group, the Society of Critical Care Medicine, and the Canadian Psychiatric Association. We will also engage with organisations that have direct contact with patients and care givers, including the Canadian Mental Health Association.

\section{Results}

A total of 423060 consecutive ICU survivors and 3081111 consecutive non-ICU hospital survivors were identified over the study period. Table 1 shows a comparison of baseline characteristics between ICU survivors and non-ICU hospital survivors. Supplemental table 3 shows year of index hospital admission. Mean age of ICU survivors was 61.7 years (standard deviation 16.5 years), and 39.1\% were women. In contrast, mean age of non-ICU hospital survivors was 53.5 years (standard deviation 20.9 years), and $64.5 \%$ were women. ICU survivors had substantial comorbid disease, with $14.7 \%$ having CCI of 3-4, and 4.8\% having CCI of 5 or higher. In particular, ICU survivors had a high prevalence of pre-existing psychiatric disease, with $15.3 \%$ having at least one previous mental health condition. When considering ICU interventions, $26.6 \%$ of survivors received invasive mechanical ventilation, $1.6 \%$ received noninvasive mechanical ventilation, and $2.4 \%$ received renal replacement therapy. Median ICU length of stay was seven days (interquartile range 4-14 days).

Among ICU survivors, 750 patients $(0.2 \%)$ died by suicide $(41.4,95 \%$ confidence interval 38.4 to 44.5 per 100000 person years during the entire follow-up period; $64.3,56.8$ to 72.8 per 100000 person years in the first year after discharge) compared with 2427 (0.1\%) non-ICU hospital survivors $(16.8,16.1$ to 17.5 per 100000 person years during the entire follow-up 


\begin{tabular}{|c|c|c|}
\hline Characteristics & ICU survivors $(n=423060)$ & Non-ICU hospital survivors $(n=3081111)$ \\
\hline Age (years), mean (SD) & $61.7(16.5)$ & $53.5(20.9)$ \\
\hline Female sex & $165348(39.1)$ & $1986193(64.5)$ \\
\hline \multicolumn{3}{|l|}{ Neighbourhood income fifth } \\
\hline Lowest & $92065(21.8)$ & $642557(20.9)$ \\
\hline Low & $88542(20.9)$ & $628244(20.4)$ \\
\hline Middle & $83548(19.7)$ & $615885(20.0)$ \\
\hline High & 80831 (19.1) & $613490(19.9)$ \\
\hline Highest & $76429(18.1)$ & $568782(18.5)$ \\
\hline Missing & $1645(0.4)$ & $12153(0.4)$ \\
\hline Rural residence & $67385(15.9)$ & $386613(12.5)$ \\
\hline Hospital length of stay (days), median (IQR) & $7(4-13)$ & $3(2-5)$ \\
\hline ICU length of stay (days), median (IQR) & $7(4-14)$ & - \\
\hline \multicolumn{3}{|l|}{ Charlson comorbidity index } \\
\hline$\leq 2$ & $340554(80.5)$ & $2858357(92.8)$ \\
\hline $3-4$ & $62109(14.7)$ & $133760(4.3)$ \\
\hline$\geq 5$ & $20397(4.8)$ & $88994(2.9)$ \\
\hline Invasive mechanical ventilation & $112481(26.6)$ & $0(0)$ \\
\hline Non-invasive mechanical ventilation & $6877(1.6)$ & $2873(0.1)$ \\
\hline Renal replacement therapy & $10159(2.4)$ & $11445(0.4)$ \\
\hline \multicolumn{3}{|l|}{ Discharge disposition } \\
\hline Home without homecare & $270170(63.9)$ & $2282408(74.1)$ \\
\hline Home with homecare & $106762(25.2)$ & $613836(19.9)$ \\
\hline Continuing care or rehabilitation & $40581(9.6)$ & $54716(1.8)$ \\
\hline Long term care facility & $5547(1.3)$ & $130151(4.2)$ \\
\hline \multicolumn{3}{|l|}{ Previous psychiatric diagnoses } \\
\hline Any psychiatric diagnosis & $64583(15.3)$ & $407299(13.2)$ \\
\hline Depression or anxiety & $61551(14.5)$ & $390607(12.7)$ \\
\hline Post-traumatic stress disorder & $1296(0.3)$ & $6946(0.2)$ \\
\hline Schizophrenia & $7012(1.7)$ & $35357(1.1)$ \\
\hline Bipolar affective disorder & $5309(1.3)$ & $26234(0.9)$ \\
\hline Hospital admissions in preceding year, mean (SD) & $0.03(0.27)$ & $0.03(0.26)$ \\
\hline Outpatient mental health visits in preceding year, mean (SD) & $1.09(5.39)$ & $0.91(4.67)$ \\
\hline Inpatient mental health admission in preceding year & $26484(0.9)$ & $8595(2.0)$ \\
\hline Suicide after hospital discharge & $750(0.2)$ & $2427(0.1)$ \\
\hline Incidence of suicide per 100000 person years $(95 \% \mathrm{Cl})$ & $41.4(38.4$ to 44.5$)$ & $16.8(16.1$ to 17.5$)$ \\
\hline Self-harm after hospital discharge & $5662(1.3)$ & $24411(0.8)$ \\
\hline Incidence of self-harm per 100000 person years $(95 \% \mathrm{Cl})$ & $327.9(319.4$ to 336.5$)$ & $177.3(175.1$ to 179.5$)$ \\
\hline Suicide or self-harm after hospital discharge & $6234(1.5)$ & $26376(0.9)$ \\
\hline Incidence of suicide or self-harm per 100000 person years $(95 \% \mathrm{Cl})$ & $361.0(352.1$ to 370.1$)$ & $191.6(189.3$ to 193.9$)$ \\
\hline
\end{tabular}

period; $24.1,22.4$ to 26.0 per 100000 person years in the first year after discharge). Self-harm was seen in $5662(1.3 \%)$ ICU survivors $(327.9,319.4$ to 336.5 per 100000 person years during the entire follow-up period; $668.5,643.1$ to 694.8 per 100000 person years in the first year after discharge), and 24411 $(0.8 \%)$ non-ICU hospital survivors $(177.3,175.1$ to 179.5 per 100000 person years during the entire follow-up period; $286.5,280.3$ to 292.8 per 100000 person years in the first year after discharge). Finally, among ICU survivors, 6234 (1.5\%) either died by suicide or had hospital visits for deliberate self-harm (361.0, 352.1 to 370.1 per 100000 person years during the entire follow-up period; $668.5,643.1$ to 694.8 per 100000 person years in the first year after discharge) compared with 26376 (0.9\%) non-ICU hospital survivors $(191.6,189.3$ to 193.9 per 100000 person years during the entire follow-up period; 286.5 , 280.3 to 292.8 per 100000 person years in the first year after discharge). After propensity score weighting (supplemental table 4), ICU admission was associated with higher risk of suicide (adjusted hazard ratio 1.22,
95\% confidence interval 1.11 to 1.33 , E value $=1.74$ ), self-harm $(1.15,1.12$ to 1.19 , E value=1.57), and the composite of suicide or self-harm $(1.15,1.12$ to 1.19, E value=1.57) compared with non-ICU hospital admission. In our evaluation of non-proportional hazards, we did not find any significant change over time (supplemental table 5).

Cumulative incidence function curves comparing risk over time between ICU survivors and non-ICU hospital survivors (fig 1) show an increased risk of suicide or self-harm in ICU survivors that is evident almost immediately after hospital discharge, and persists for years afterwards. A similar relation is observed when patients are stratified by pre-existing mental health history.

Table 2 shows assessment of differences between ICU survivors who died by suicide or had self-harm events versus those who did not. ICU survivors who died by suicide or had self-harm events tended to be younger, live in poorer neighbourhoods, have lower comorbidity burden, and be discharged directly home from the hospital. A higher prevalence of pre-existing 
ICU status

Non-ICU hospital survivors

--- ICU survivors
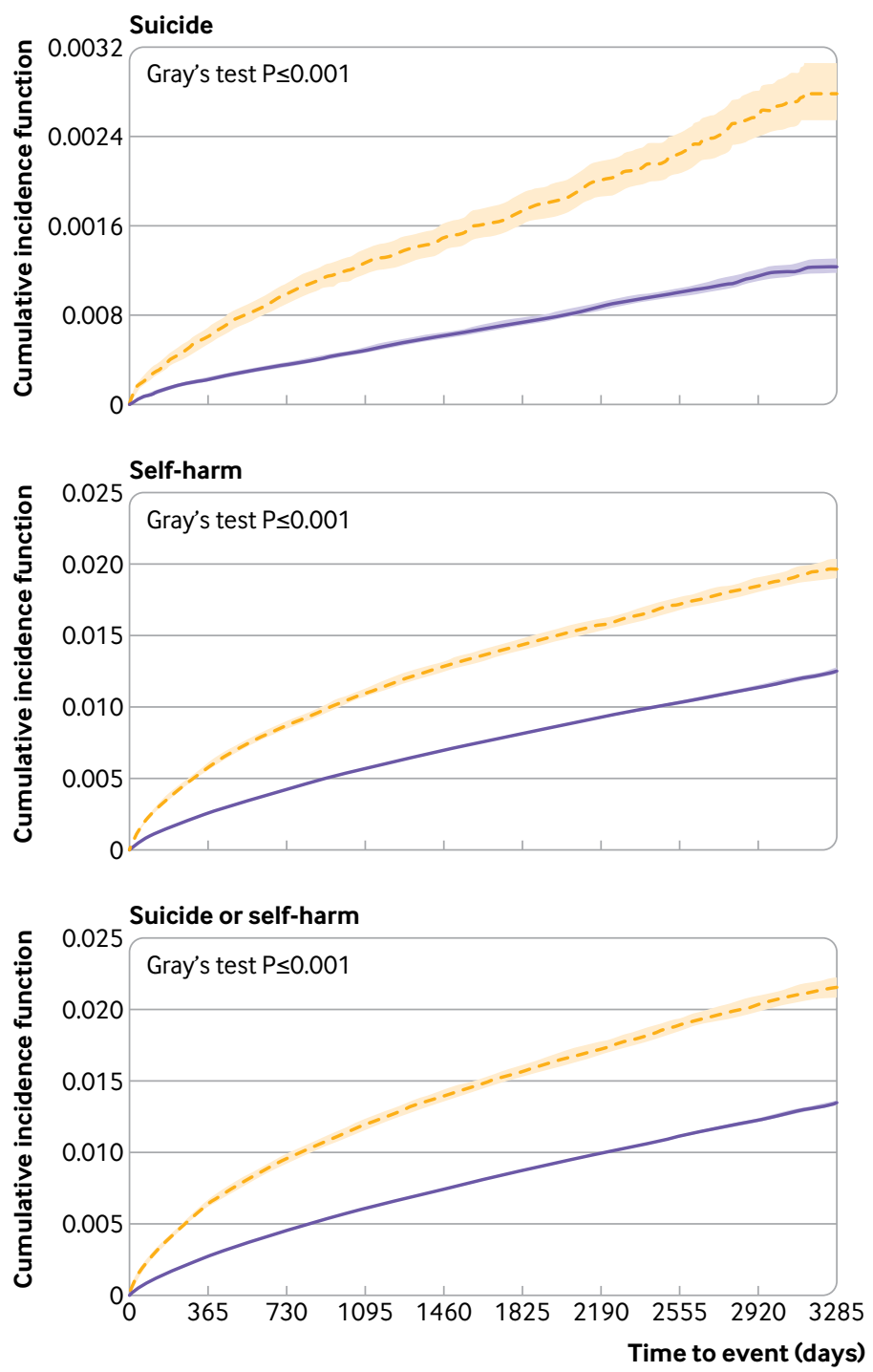

ICU and pre-existing mental health status

---- Non-ICU hospital survivors without pre-existing mental health diagnoses

-- - ICU survivors without pre-existing mental health diagnoses

- - Non-ICU hospital survivors with pre-existing mental health diagnoses

- - ICU survivors with pre-existing mental health diagnoses
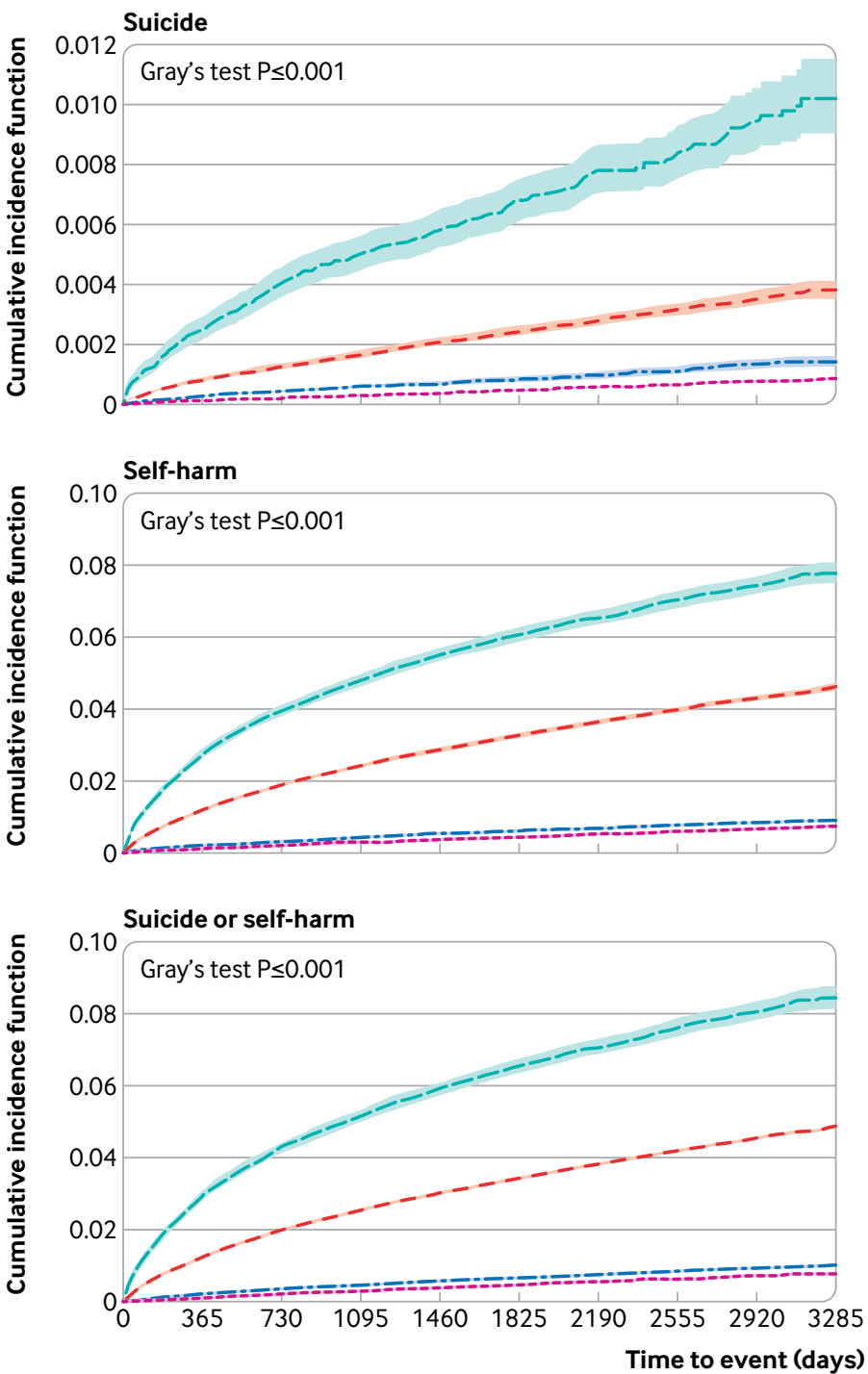

Fig 1 | Left panels: cumulative incidence function curves for suicide, self-harm, and suicide or self-harm among ICU survivors and non-ICU hospital survivors. Right panels: cumulative incidence function curves for suicide, self-harm, and suicide or self-harm among ICU survivors with or without pre-existing mental health diagnoses, and non-ICU hospital survivors with or without pre-existing mental health diagnoses

mental health diagnoses was also found among patients who died by suicide or had self-harm events.

We further evaluated prognostic factors associated with suicide, self-harm, or the composite outcome among ICU survivors (table 3). Previous mental health diagnoses were strongly associated with increased prognosis for either suicide or self-harm, including depression or anxiety (hazard ratio 5.69, 95\% confidence interval 5.38 to 6.02$)$, PTSD $(1.87,1.64$ to 2.13), schizophrenia (1.39, 1.28 to 1.52$)$ and bipolar disorder $(2.38,2.20$ to 2.58$)$. The use of invasive mechanical ventilation $(1.45,1.38$ to 1.54$)$ and renal replacement therapy $(1.35,1.17$ to 1.56$)$ were also associated with prognosis for suicide or self-harm, as was a history of a previous hospital admission (1.25, 1.19 to 1.30 per previous admission). Factors associated with lower prognostic risk for suicide or self-harm included increasing age (0.96, 0.96 to 0.96 per one year older), residing in the highest income fifth relative to the lowest $(0.67,0.62$ to 0.73$)$, and discharge to a continuing care or rehabilitation facility $(0.80,0.70$ to 0.90$)$ or long term care facility $(0.29,0.15$ to 0.57$)$ compared with discharge home independently. 


\begin{tabular}{|c|c|c|c|c|}
\hline Characteristics & $\begin{array}{l}\text { Neither suicide nor } \\
\text { self-harm }(n=416826)\end{array}$ & Suicide $(n=750)$ & Self-harm $(n=5484)$ & $\begin{array}{l}\text { Suicide or } \\
\text { self-harm }(n=6234)\end{array}$ \\
\hline Age (years), mean (SD) & $62.0(16.4)$ & $50.9(16.0)$ & $44.2(16.0)$ & $45.0(16.0)$ \\
\hline Female sex & $162595(39.0)$ & $228(30.4)$ & $2525(46.0)$ & $2753(44.2)$ \\
\hline \multicolumn{5}{|l|}{ Neighbourhood income fifth } \\
\hline Lowest & $90089(21.6)$ & $187(24.9)$ & $1789(32.6)$ & $1976(31.7)$ \\
\hline Low & $87214(20.9)$ & $181(24.1)$ & $1147(20.9)$ & $1328(21.3)$ \\
\hline Middle & $82430(19.8)$ & $153(20.4)$ & $965(17.6)$ & $1118(17.9)$ \\
\hline High & $79880(19.2)$ & $124(16.5)$ & $827(15.1)$ & $951(15.3)$ \\
\hline Highest & $75617(18.1)$ & $101(13.5)$ & $711(13.0)$ & $812(13.0)$ \\
\hline Missing & $1596(0.4)$ & $4(0.5)$ & $45(0.8)$ & $49(0.8)$ \\
\hline Rural residence & $66499(16.0)$ & $121(16.1)$ & $765(13.9)$ & $886(14.2)$ \\
\hline Hospital length of stay (days), median (IQR) & $7(4-13)$ & $5(3-10)$ & $4(3-9)$ & $4(3-9)$ \\
\hline ICU length of stay, (days), median (IQR) & $7(4-14)$ & $7(4-14)$ & $7(3-13)$ & $7(4-13)$ \\
\hline \multicolumn{5}{|l|}{ Charlson comorbidity index } \\
\hline$\leq 2$ & $334780(80.3)$ & $693(92.4)$ & $5081(92.7)$ & $5774(92.6)$ \\
\hline $3-4$ & $61736(14.8)$ & $40(5.3)$ & $332(6.1)$ & $372(6.0)$ \\
\hline$\geq 5$ & $20309(4.9)$ & $17(2.3)$ & $71(1.3)$ & $88(1.4)$ \\
\hline Invasive mechanical ventilation & $110345(26.5)$ & $270(36.0)$ & $1866(34.0)$ & $2136(34.3)$ \\
\hline Non-invasive mechanical ventilation & $6801(1.6)$ & $11(1.5)$ & $65(1.2)$ & $76(1.2)$ \\
\hline Renal replacement therapy & $9953(2.4)$ & $27(3.6)$ & $179(3.3)$ & $206(3.3)$ \\
\hline \multicolumn{5}{|l|}{ Discharge disposition } \\
\hline Home without homecare & $265241(63.6)$ & $574(76.5)$ & $4355(79.4)$ & $4929(79.1)$ \\
\hline Home with homecare & $105804(25.4)$ & $122(16.3)$ & $836(15.2)$ & $958(15.4)$ \\
\hline Continuing care or rehabilitation & $40244(9.7)$ & $54(7.2)$ & $283(5.2)$ & $337(5.4)$ \\
\hline Long term care facility & $5537(1.3)$ & $0(0)$ & $10(0.2)$ & $10(0.2)$ \\
\hline \multicolumn{5}{|l|}{ Previous psychiatric diagnoses } \\
\hline Any psychiatric diagnosis & $60640(14.5)$ & $439(58.5)$ & $3504(63.9)$ & $3943(63.2)$ \\
\hline Depression or anxiety & $57773(13.9)$ & $423(56.4)$ & $3355(61.2)$ & $3778(60.6)$ \\
\hline Post-traumatic stress disorder & $1040(0.2)$ & $19(2.5)$ & $237(4.3)$ & $256(4.1)$ \\
\hline Schizophrenia & $6303(1.5)$ & $83(11.1)$ & $626(11.4)$ & $709(11.4)$ \\
\hline Bipolar affective disorder & $4394(1.1)$ & $91(12.1)$ & $824(15.0)$ & $915(14.7)$ \\
\hline Hospital admissions in preceding year, mean (SD) & $0.03(0.27)$ & $0.03(0.25)$ & $0.10(0.55)$ & $0.09(0.52)$ \\
\hline Outpatient mental health visits in preceding year, mean (SD) & $0.98(5.00)$ & $5.63(11.15)$ & $8.87(15.95)$ & $8.48(15.48)$ \\
\hline Inpatient mental health admission in preceding year & $6680(1.6)$ & $243(32.4)$ & $1672(30.5)$ & $1915(30.7)$ \\
\hline
\end{tabular}

Finally, we evaluated crude incidence of suicide, self-harm, or the composite outcome among prespecified subgroups of interest, and depicted this relation through the use of heat maps, graphically showing the subgroups with the highest incidence of these outcomes (fig 2). The highest crude incidence of these outcomes is observed in younger patients (age 18-34), with pre-existing mental health diagnoses, and receiving invasive mechanical ventilation or renal replacement therapy. Supplemental table 6 shows further stratification by sex.

\section{Discussion}

\section{Principal findings}

We used population based data across nine years from the province of Ontario, Canada to evaluate the incidence and prognostic factors for suicide and selfharm among 423060 consecutive survivors of critical illness. We compared incidence in ICU survivors and hospital survivors who did not require ICU admission through propensity score weighting. These analyses showed that survival after ICU admission was associated with suicide and self-harm compared with non-ICU hospital survivors, even after propensity score weighting. Among ICU survivors, prognostic factors associated with suicide or self-harm included previous history of mental health diagnoses (specifically depression or anxiety, PTSD, schizophrenia, and bipolar disorder) and receipt of invasive mechanical ventilation or renal replacement therapy in the ICU. Therefore, ICU survivorship is associated with future suicide and deliberate self-harm, with identifiable prognostic factors for consideration in future health policy and care planning.

\section{Strengths and limitations of study}

Our study has relevant strengths and limitations. In terms of strengths, our study evaluates a new and important research question, and includes a large cohort of consecutive ICU survivors from an entire population, with minimal missing data. We adhered closely to reporting guidelines and used statistical methods to limit confounding and enhance trustworthiness in measures of association.

However, our study has important limitations. We applied stringent methods to account for differences in patient characteristics, particularly with relation to previous mental health history among patients (including previous psychiatric diagnoses, previous outpatient mental health provider visits, and previous history of self-harm behaviours requiring hospital attention). However, given the observational study 


\begin{tabular}{|c|c|c|c|}
\hline Factors & Suicide & Self-harm & Suicide or self-harm \\
\hline Age (per one year) & $0.98(0.98$ to 0.99$)$ & $0.96(0.95$ to 0.96$)$ & $0.96(0.96$ to 0.96$)$ \\
\hline Female sex & 0.57 (0.49 to 0.67$)$ & $1.00(1.02$ to 1.14$)$ & $1.00(0.95$ to 1.06$)$ \\
\hline \multicolumn{4}{|l|}{ Neighbourhood income fifth } \\
\hline Lowest & Reference & Reference & Reference \\
\hline Low & $1.13(0.92$ to 1.39$)$ & $0.83(0.75$ to 0.87$)$ & $0.83(0.77$ to 0.89$)$ \\
\hline Middle & $1.09(0.87$ to 1.35$)$ & $0.78(0.70$ to 0.82$)$ & $0.78(0.73$ to 0.84$)$ \\
\hline High & $0.92(0.73$ to 1.16$)$ & $0.71(0.63$ to 0.74$)$ & $0.71(0.65$ to 0.76$)$ \\
\hline Highest & $0.82(0.64$ to 1.05$)$ & $0.67(0.61$ to 0.72$)$ & $0.67(0.62$ to 0.73$)$ \\
\hline Urban residence & $0.92(0.75$ to 1.13$)$ & $1.05(0.99$ to 1.15$)$ & $1.05(0.98$ to 1.13$)$ \\
\hline Hospital length of stay (per one day) & 0.98 (0.97 to 0.99) & $0.99(0.99$ to 1.00$)$ & $0.99(0.99$ to 1.00$)$ \\
\hline ICU length of stay (per one day) & $1.00(1.00$ to 1.01$)$ & $1.00(1.00$ to 1.00$)$ & $1.00(1.00$ to 1.00$)$ \\
\hline \multicolumn{4}{|l|}{ Charlson comorbidity index } \\
\hline$\leq 2$ & Reference & Reference & Reference \\
\hline $3-4$ & $0.50(0.36$ to 0.70$)$ & $0.73(0.68$ to 0.85$)$ & $0.73(0.65$ to 0.81$)$ \\
\hline$\geq 5$ & $1.07(0.65$ to 1.74$)$ & $0.76(0.55$ to 0.88$)$ & $0.76(0.61$ to 0.94$)$ \\
\hline Invasive mechanical ventilation & $1.50(1.28$ to 1.77$)$ & $1.45(1.37$ to 1.53$)$ & $1.45(1.38$ to 1.54$)$ \\
\hline Renal replacement therapy & $1.56(1.05$ to 2.32$)$ & $1.35(1.16$ to 1.56$)$ & $1.35(1.17$ to 1.56$)$ \\
\hline \multicolumn{4}{|l|}{ Discharge disposition } \\
\hline Home without homecare & Reference & Reference & Reference \\
\hline Home with homecare & $0.87(0.71$ to 1.08$)$ & $0.82(0.75$ to 0.88$)$ & $0.82(0.76$ to 0.88$)$ \\
\hline Continuing care or rehabilitation & $1.05(0.75$ to 1.48$)$ & $0.79(0.67$ to 0.89$)$ & $0.79(0.70$ to 0.91$)$ \\
\hline Long term care facility & $-^{\star}$ & $0.29(0.17$ to 0.63$)$ & $0.29(0.15$ to 0.57$)$ \\
\hline \multicolumn{4}{|l|}{ Psychiatric diagnosest } \\
\hline Depression or anxiety & $5.73(4.87$ to 6.73$)$ & $5.69(5.38$ to 6.05$)$ & $5.69(5.38$ to 6.02$)$ \\
\hline Post-traumatic stress disorder & $1.50(0.93$ to 2.41$)$ & 1.87 (1.67 to 2.19$)$ & $1.87(1.64$ to 2.13$)$ \\
\hline Schizophrenia & 1.51 (1.16 to 1.96$)$ & $1.39(1.28$ to 1.53$)$ & 1.39 (1.28 to 1.52$)$ \\
\hline Bipolar affective disorder & $2.20(1.70$ to 2.84$)$ & 2.38 (2.19 to 2.59$)$ & $2.38(2.20$ to 2.58$)$ \\
\hline Hospital admissions in preceding year (per previous admission) & $0.88(0.66$ to 1.18$)$ & $1.26(1.20$ to 1.31$)$ & $1.25(1.19$ to 1.30$)$ \\
\hline Outpatient psychiatric visits in preceding year (per previous visit) & $1.02(1.01$ to 1.03$)$ & $1.02(1.02$ to 1.02$)$ & $1.02(1.02$ to 1.02$)$ \\
\hline \multicolumn{4}{|l|}{ Year of index hospital admission } \\
\hline 2009 & $0.57(0.36$ to 0.92$)$ & $1.36(1.12$ to 1.65$)$ & $1.25(1.04$ to 1.49$)$ \\
\hline 2010 & $0.54(0.33$ to 0.87$)$ & $1.19(0.98$ to 1.45$)$ & $1.10(0.92$ to 1.32$)$ \\
\hline 2011 & $0.53(0.32$ to 0.86$)$ & $1.09(0.89$ to 1.32$)$ & $1.02(0.84$ to 1.22$)$ \\
\hline 2012 & $0.68(0.42$ to 1.10$)$ & $1.01(0.82$ to 1.23$)$ & $0.96(0.79$ to 1.15$)$ \\
\hline 2013 & $0.57(0.35$ to 0.94$)$ & $1.03(0.84$ to 1.26$)$ & $0.97(0.80$ to 1.17$)$ \\
\hline 2014 & $0.75(0.46$ to 1.23$)$ & $0.98(0.79$ to 1.20$)$ & $0.94(0.78$ to 1.14$)$ \\
\hline 2015 & $0.63(0.37$ to 1.05$)$ & $0.97(0.79$ to 1.20$)$ & $0.93(0.76$ to 1.13$)$ \\
\hline 2016 & $0.91(0.54$ to 1.52$)$ & $0.98(0.79$ to 1.22$)$ & $0.96(0.78$ to 1.18$)$ \\
\hline 2017 & Reference & Reference & Reference \\
\hline
\end{tabular}

design, we cannot rule out residual confounding, and therefore interpretation of our results must account for this limitation. Similarly, while we followed the PROGRESS guidelines in the creation of our prognostic model, ${ }^{33} 34$ we were limited by the retrospective nature of our dataset, and at present, a lack of external validation. As such, the associations we identified require further study. Additionally, we were limited by the granularity of the available data, such as initial ICU admission diagnoses, severity of illness indices, drugs received in hospital (eg, vasoactive agents), and mechanism of suicide. Moreover, the self-harm outcome was derived as a composite of various ICD codes that have not been validated with chart review (and therefore could result in misclassification), and only included deliberate self-harm behaviours that prompted medical attention. However, we conducted separate analyses of self-harm and suicide, and showed similar findings, which might reduce concerns about the measure of self-harm as a study outcome.
Finally, we have few data on the association between various protective factors (eg, personality traits, support system, receipt of specific post-discharge psychological treatments), and suicide or self-harm. Future research should seek to understand if such interventions might attenuate the prognosis for suicide and self-harm in these patients.

\section{Comparison with other studies}

Psychological morbidity is common among ICU survivors, and is often attributed to physical manifestations of post-intensive care syndrome, ${ }^{12}$ such as potentially long lasting muscle weakness, reduced exercise capacity, cognitive impairments, fatigue, chronic pain, substance misuse, and financial hardship..$^{4-812-1536}$ However, our work shows that ICU survivors who go on to complete suicide and self-harm are markedly different from the patients with chronic physical morbidity after discharge. These patients tend to be younger, have previous mental health 


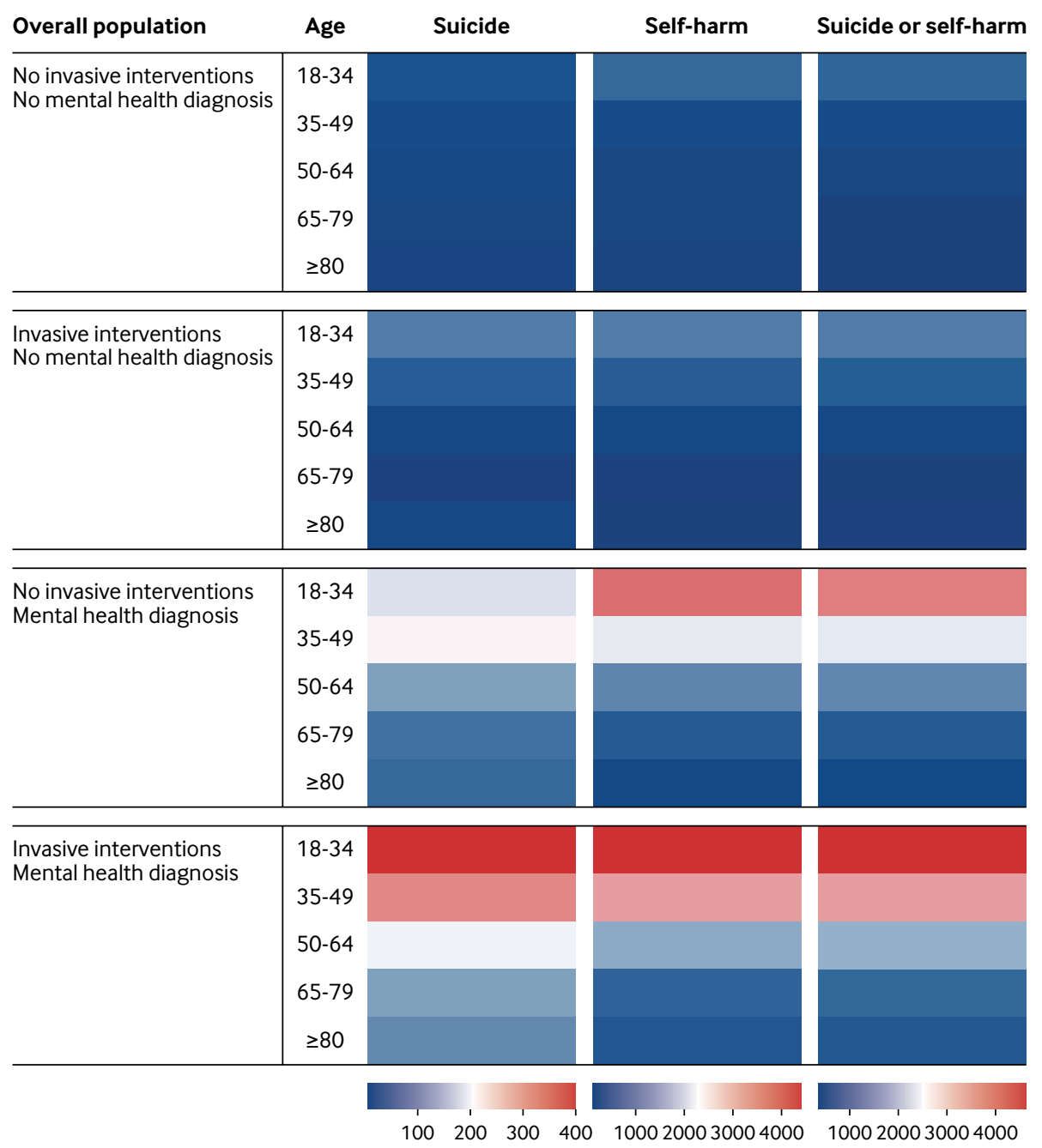

Fig 2 | Heat maps showing crude incidence of suicide, self-harm, and suicide or self-harm per 100000 person years, stratified by age, receipt of invasive interventions (invasive mechanical ventilation or renal replacement therapy), and previous mental health diagnoses

history but otherwise lower comorbidity burden, and also seem to maintain some degree of physical independence at discharge (on the basis of a lower proportion of discharge to long term care). As a result, the population of ICU survivors at risk of future suicide and self-harm seems to be distinct from the population that we consistently associate with post-intensive care syndrome, ${ }^{5}$ and this was one of the most important findings in our study. Identification of unique at risk ICU survivor groups is the cornerstone in the derivation of methods for mitigation of adverse long term outcomes among these patients. ${ }^{4}$

We found that previous mental health diagnoses had the strongest prognostic association with future suicide and self-harm, consistent with existing studies in a variety of populations. ${ }^{17}$ This finding highlights the importance of assessing for such morbidity among ICU survivors, and several tools for screening in hospital exist. ${ }^{12}$ Receipt of invasive mechanical ventilation and renal replacement therapy were prognostic factors that were significantly associated with self-harm and suicide. These life support interventions, particularly when used over a prolonged duration, have also been associated with a higher incidence of mood disorders. ${ }^{1314}$

We found a higher incidence of suicide and self-harm among ICU survivors in lower income neighbourhoods. An inverse association between suicide and socioeconomic status has been previously shown, and could relate to higher concomitant comorbidities, mental health diagnoses, and reduced access to mental health resources. ${ }^{37}$ Finally, suicide and self-harm appeared to be higher among patients discharged home independently compared with those discharged to a healthcare facility. Again, this is in contrast to the population typically associated with post-intensive care syndrome. ${ }^{5}$ This prognostic finding might be related to reduced assistance with activities of daily living, lack of emotional and social support, and reduced access to healthcare. Furthermore, patients discharged to these long term care facilities might have reduced function and cognitive impairment, with reduced capacity, which could also account for the lower incidence of suicide and self-harm. ${ }^{38}$ 
While the overall magnitude of risk of suicide among ICU survivors (compared with non-ICU hospital survivors) does not appear to be substantial, it might have important implications at the population level. Firstly, determination of post-discharge suicide risk among patients admitted to hospital is complex, ${ }^{17}$ and thus further identification of possible prognostic factors has value. Secondly, while all ICU survivors might not be equally affected, there could be particular subpopulations at heightened risk. Therefore, we stratified patients by age, previous mental health diagnoses, and ICU treatment interventions to identify varying prognosis levels across relevant and readily identifiable patient subgroups. Younger age, pre-existing mental health disease, and life support treatments in ICU were associated with higher prognosis for suicide and self-harm. Identification of patients at high risk could allow for informed targeting of preventative strategies. Our work further emphasises that differences in incidence of suicide and self-harm between ICU survivors and non-ICU hospital survivors develop early after hospital discharge, and are sustained over subsequent years. ${ }^{5}$ Therefore, early intervention might be particularly important. Focus must be on the assessment of high risk patient populations, and identifying individual patients who could benefit from further assessment and treatment. ${ }^{39}$ Patient follow-up is of potential importance, and additional investigation into outpatient care of ICU survivors (particularly in relation to mental health and palliative care) after discharge represents an important avenue for future research. Post-intensive care follow-up clinics could be used to facilitate further identification and treatment of patients with mental health disease after an ICU admission, ${ }^{40}$ and could involve consultants in psychiatry or psychology. Additionally, primary care providers have a fundamental role in assessing and evaluating these patients after ICU and hospital discharge, and early follow-up should be considered. ${ }^{41}$

\section{Conclusion and policy implications}

In this large population based study from Ontario, Canada, survival from critical illness was associated with subsequent suicide and self-harm, with ICU survivors having a higher incidence of these outcomes compared with hospital survivors who never required ICU admission. Prognostic factors associated with suicide and self-harm among ICU survivors included pre-existing psychiatric illness, lower socioeconomic status, life support interventions in the ICU, and hospital discharge to home rather than a healthcare facility. Future research should identify methods of reducing suicide and self-harm in ICU survivors, particularly those with these additional prognostic factors.

AUTHOR AFFILIATIONS

${ }^{1}$ Division of Critical Care, Department of Medicine, University of Ottawa, Ottawa, ON, Canada

${ }^{2}$ Department of Emergency Medicine, University of Ottawa, Ottawa, ON, Canada
${ }^{3}$ ICES, Toronto, ON, Canada

${ }^{4}$ Clinical Epidemiology Program, Ottawa Hospital Research Institute, Ottawa, ON, Canada

${ }^{5}$ School of Epidemiology and Public Health, University of Ottawa, Ottawa, ON, Canada

${ }^{6}$ Bruyère Research Institute, Ottawa, ON, Canada

${ }^{7}$ Division of Nephrology, Department of Medicine, University of Ottawa, Ottawa, ON, Canada

${ }^{8}$ Department of Family Medicine, University of Ottawa, Ottawa, ON, Canada

${ }^{9}$ Interdepartmental Division of Critical Care Medicine, University of Toronto, Toronto, ON, Canada

${ }^{10}$ Toronto General Hospital Research Institute, University Health Network, Toronto, ON, Canada

${ }^{11}$ Institute of Health Policy, Management and Evaluation, Dalla Lana School of Public Health, University of Toronto, Toronto, ON, Canada

${ }^{12}$ Department of Medicine, Division of Pulmonary and Critical Care Medicine, Johns Hopkins University School of Medicine, Baltimore, MD, USA

${ }^{13}$ Department of Physical Medicine and Rehabilitation, Johns Hopkins University School of Medicine, Baltimore, MD, USA

${ }^{14}$ Department of Medicine, Division of Critical Care, McMaster University, Hamilton, ON, Canada

${ }^{15}$ Department of Health Research Methods, Evidence, and Impact, McMaster University, Hamilton, ON, Canada

${ }^{16}$ Department of Critical Care Medicine, Sunnybrook Health Sciences Centre, Toronto, ON, Canada

${ }^{17} \mathrm{Li}$ Ka Shing Knowledge Institute, St Michael's Hospital, Toronto, ON, Canada

${ }^{18}$ Department of Psychiatry and Behavioural Sciences, Johns Hopkins University School of Medicine, Baltimore, MD, USA

${ }^{19}$ Intensive Care National Audit and Research Centre, Napier House, London, UK

${ }^{20}$ Department of Critical Care, Queensway Carleton Hospital, Ottawa, ON, Canada

${ }^{21}$ Division of Palliative Care, Department of Medicine, University of Ottawa, Ottawa, ON, Canada

${ }^{22}$ Institut du Savoir Montfort, Ottawa, ON, Canada

Contributors: SMF, DQ, MMS, MK, PT, and KK conceived the study idea. All authors participated in study design. MP and RT gathered the data and performed data analyses. All authors interpreted the data analyses. All authors cowrote and revised the manuscript for intellectual content. All authors provided their final approval for manuscript submission. All authors agree to be accountable for all aspects of the work. PT and KK contributed equally as cosenior authors. SMF is the guarantor of the study. The corresponding author attests that all listed authors meet authorship criteria and that no others meeting the criteria have been omitted.

Funding: Funded by the Institut du Savoir Montfort, Hôpital Montfort, Ottawa (Canada). This study was supported by ICES, which is funded by an annual grant from the Ontario Ministry of Health and Long-term Care (MOHLTC). The opinions, results, and conclusions reported in this paper are those of the authors and are independent from the funding sources. No endorsement by ICES or the Ontario MOHLTC is intended or should be inferred. Parts of this material are based on data or information compiled and provided by the Canadian Institute for Health Information (CIHI). However, the analyses, conclusions, and opinions and statements expressed in the material are those of the authors, and not necessarily those of $\mathrm{CIHI}$.

Competing interests: All authors have completed the ICMJE uniform disclosure form at www.icmje.org/coi disclosure.pdf and declare: support from the Institut du Savoir Montfort, Hôpital Montfort, Ontario MOHLTC, CIHI for the submitted work; MMS reports Continuing Medical Education funding from AstraZeneca, and is supported by the Jindal Research Chair in the Prevention of Kidney Disease at the University of Ottawa; DMN reports financial or in-kind support for consultancy or research (outside the scope of this research) from Haisco-USA Pharmaceuticals, GlaxoSmithKline (UK), Novartis Pharma (Switzerland), Baxter Healthcare Corporation, and Reck Medical Devices; BR is supported by a Hamilton Health Sciences new investigator grant; DJC is supported by a Canada Research Chair in Critical Care Knowledge Translation; HW is supported by a Canada Research Chair in Critical Care Organisation and Outcomes; 
RAF is the $\mathrm{H}$ Barrie Fairley Professor of Critical Care at the University Health Network and University of Toronto Interdepartmental Division of Critical Care Medicine; PT is supported by a Physician Services Incorporated Graham Farquharson Knowledge Translation Fellowship; all other authors declare no support from any organisation for the submitted work; no financial relationships with any organisations that might have an interest in the submitted work in the previous three years; and no other relationships or activities that could appear to have influenced the submitted work.

Ethical approval: Studies conducted at ICES using administrative data fall under section 45 of the Personal Health Information Protection Act of Ontario, and as such, do not require research ethics board approval.

Data sharing: The dataset from this study is held securely in coded form at ICES. While data sharing agreements prohibit ICES from making the dataset publicly available, access might be granted to those who meet prespecified criteria for confidential access (available at www.ices.on.ca/DAS). The full dataset creation plan and underlying analytic code are available from the authors upon request, understanding that the computer programs might rely upon coding templates or macros that are unique to ICES and are therefore either inaccessible or could require modification.

The corresponding author (SMF) affirms that the manuscript is an honest, accurate, and transparent account of the study being reported; that no important aspects of the study have been omitted; and that any discrepancies from the study as planned have been explained.

Dissemination to participants and related patient and public communities: Because participants cannot be identified from our anonymised and deidentified study data source, we are unable to directly disseminate results of the study to its participants. However, we intend to disseminate our study findings through a variety of mediums, including presentation at local and national critical care conferences, memorandums through important organisations related to critical care and psychiatry, social media (namely Twitter), institutional press releases (Ottawa Hospital Research Institute, University of Ottawa), and podcasts (Dr Kwadwo Kyeremanteng is the host of the Solving Healthcare podcast, which has a broad national audience). We will also engage with local and national news media to disseminate these findings through news outlets.

Provenance and peer review: Not commissioned; externally peer reviewed.

This is an Open Access article distributed in accordance with the Creative Commons Attribution Non Commercial (CC BY-NC 4.0) license, which permits others to distribute, remix, adapt, build upon this work non-commercially, and license their derivative works on different terms, provided the original work is properly cited and the use is noncommercial. See: http://creativecommons.org/licenses/by-nc/4.0/.

1 Adhikari NK, Fowler RA, Bhagwanjee S, Rubenfeld GD. Critical care and the global burden of critical illness in adults. Lancet 2010;376:133946. doi:10.1016/S0140-6736(10)60446-1

2 Zimmerman JE, Kramer AA, Knaus WA. Changes in hospital mortality for United States intensive care unit admissions from 1988 to 2012. Crit Care 2013;17:R81. doi:10.1186/cc12695

3 Flaatten H, Kvåle R. Survival and quality of life 12 years after ICU. A comparison with the general Norwegian population. Intensive Care Med 2001:27:1005-11. doi:10.1007/s001340100960

4 Needham DM, Davidson J, Cohen H, et al. Improving long-term outcomes after discharge from intensive care unit: report from a stakeholders' conference. Crit Care Med 2012;40:502-9. doi:10.1097/CCM.0b013e318232da75

5 Azoulay E, Vincent JL, Angus DC, et al. Recovery after critical illness: putting the puzzle together-a consensus of 29. Crit Care 2017:21:296 doi:10.1186/s13054-017-1887-7

6 Herridge MS, Cheung AM, Tansey CM, et al, Canadian Critical Care Trials Group. One-year outcomes in survivors of the acute respiratory distress syndrome. N Engl/ Med 2003:348:683-93. doi:10.1056/ NEJMoaO22450

7 Herridge MS, Tansey CM, Matté A, et al, Canadian Critical Care Trials Group. Functional disability 5 years after acute respiratory distress syndrome. N Engl J Med 2011;364:1293-304. doi:10.1056/ NEJMoa1011802

8 Pandharipande PP, Girard TD, Jackson IC, et al, BRAIN-ICU Study Investigators. Long-term cognitive impairment after critical illness. N Engl J Med 2013;369:1306-16. doi:10.1056/NEJMoa1301372

9 Neufeld KJ, Leoutsakos JS, Yan H, et al. Fatigue symptoms during the first year Following ARDS. Chest 2020;158:999-1007. doi:10.1016/j.chest.2020.03.059
10 Oeyen SG, Vandijck DM, Benoit DD, Annemans L, Decruyenaere IM. Quality of life after intensive care: a systematic review of the literature. Crit Care Med 2010;38:2386-400. doi:10.1097/ CCM.0b013e3181f3dec5

11 Brooks R, Kerridge R, Hillman K, Bauman A, Daffurn K. Quality of life outcomes after intensive care. Comparison with a community group. Intensive Care Med 1997;23:581-6. doi:10.1007/s001340050376

12 Prince E, Gerstenblith TA, Davydow D, Bienvenu OJ. Psychiatric morbidity after critical illness. Crit Care Clin 2018:34:599-608. doi:10.1016/j.ccc.2018.06.006

13 Wunsch $\mathrm{H}$, Christiansen CF, Johansen MB, et al. Psychiatric diagnoses and psychoactive medication use among nonsurgical critically ill patients receiving mechanical ventilation. JAMA 2014;311:1133-42. doi:10.1001/jama.2014.2137

14 Sivanathan L, Wunsch H, Vigod S, Hill A, Pinto R, Scales DC. Mental illness after admission to an intensive care unit. Intensive Care Med 2019;45:1550-8. doi:10.1007/s00134-019-05752-5

15 Sareen J, Olafson K, Kredentser MS, et al. The 5-year incidence of mental disorders in a population-based ICU survivor cohort. Crit Care Med 2020;48:e675-83. doi:10.1097/CCM.0000000000004413

16 Sisti DA, Joffe S. Implications of zero suicide for suicide prevention research. JAMA 2018:320:1633-4. doi:10.1001/jama.2018.13083

17 Turecki G, Brent DA, Gunnell D, et al. Suicide and suicide risk. Nat Rev Dis Primers 2019;5:74. doi:10.1038/s41572-019-0121-0

18 Roberts DJ, Nagpal SK, Kubelik D, et al. Association between neuraxial anaesthesia or general anaesthesia for lower limb revascularisation surgery in adults and clinical outcomes: population based comparative effectiveness study. BMJ 2020;371:m4104. doi:10.1136/bmj.m4104

19 Jha P, Deboer D, Sykora K, Naylor CD. Characteristics and mortality outcomes of thrombolysis trial participants and nonparticipants: a population-based comparison. J Am Coll Cardiol 1996;27:1335-42. doi:10.1016/0735-1097(96)00018-6

20 Scales DC, Guan J, Martin CM, Redelmeier DA. Administrative data accurately identified intensive care unit admissions in Ontario. J Clin Epidemiol 2006:59:802-7. doi:10.1016/i.jclinepi.2005.11.015

21 Fernando SM, Qureshi D, Tanuseputro P, et al. Mortality and costs following extracorporeal membrane oxygenation in critically ill adults: a population-based cohort study. Intensive Care Med 2019;45:15809. doi:10.1007/s00134-019-05766-z

22 Charlson ME, Pompei P, Ales KL, MacKenzie CR. A new method of classifying prognostic comorbidity in longitudinal studies: development and validation. J Chronic Dis 1987;40:373-83. doi:10.1016/0021-9681(87)90171-8

23 Sundararajan V, Henderson T, Perry C, Muggivan A, Quan H, Ghali WA. New ICD-10 version of the Charlson comorbidity index predicted in-hospital mortality. J Clin Epidemiol 2004;57:1288-94. doi:10.1016/j.jclinepi.2004.03.012

24 Walkup JT, Townsend L, Crystal S, Olfson M. A systematic review of validated methods for identifying suicide or suicidal ideation using administrative or claims data. Pharmacoepidemiol Drug Saf 2012;21(Suppl 1):174-82. doi:10.1002/pds.2335

25 Chan MK, Bhatti H, Meader N, et al. Predicting suicide following self-harm: systematic review of risk factors and risk scales. $\mathrm{Br}$ J Psychiatry 2016;209:277-83. doi:10.1192/bjp.bp.115.170050

26 Gatov E, Kurdyak P, Sinyor M, Holder L, Schaffer A. Comparison of vital statistics definitions of suicide against a coroner reference standard: a population-based linkage study. Can J Psychiatry 2018;63:152-60. doi:10.1177/0706743717737033

27 Sood MM, Rhodes E, Talarico R, et al. Suicide, suicide attempts and self-harm among medical residents and physicians in Ontario. CMAJ 2021.

28 Amartey A Chiu M, Gatov E, et al. Mental Health and Addictions System Performance in Ontario: A Baseline Scorecard. Technical Appendix https://www.ices.on.ca/ /media/Files/AtlasesReports/2018/MHASEF/Technical-Appendix.ashx?la=en-CA2018

29 Bayoumi I, Schultz SE, Glazier RH. Primary care reform and funding equity for mental health disorders in Ontario: a retrospective observational population-based study. CMAI Open 2020;8:E455-61. doi:10.9778/cmajo.20190153

30 Lederer DJ, Bell SC, Branson RD, et al. Control of confounding and reporting of results in causal inference studies. Guidance for authors from editors of respiratory, sleep, and critical care journals. Ann Am Thorac Soc 2019;16:22-8. doi:10.1513/AnnalsATS.201808-564PS

31 Thomas LE, Li F, Pencina MJ. Overlap weighting: a propensity score method that mimics attributes of a randomized clinical trial. JAMA 2020;323:2417-8. doi:10.1001/jama.2020.7819

32 VanderWeele TJ, Ding P. Sensitivity analysis in observational research: introducing the E-value. Ann Intern Med 2017;167:268-74. doi:10.7326/M16-2607

33 Riley RD, Hayden JA, Steyerberg EW, et al, PROGRESS Group. Prognosis Research Strategy (PROGRESS) 2: prognostic factor research. PLoS Med 2013;10:e1001380. doi:10.1371/journal. pmed. 1001380 
34 Steyerberg EW, Moons KG, van der Windt DA, et al, PROGRESS Group. Prognosis Research Strategy (PROGRESS) 3: prognostic model research. PLoS Med 2013;10:e1001381. doi:10.1371/journal.pmed.1001381

35 Leisman DE, Harhay MO, Lederer DJ, et al. Development and reporting of prediction models: guidance for authors from editors of respiratory, sleep, and critical care journals. Crit Care Med 2020;48:623-33. doi:10.1097/CCM.0000000000004246

36 Wunsch H, Hill AD, Fu L, et al. New opioid use after invasive mechanical ventilation and hospital discharge. Am / Respir Crit Care Med 2020;202:568-75. doi:10.1164/rccm.201912-25030C

37 Lewis G, Sloggett A. Suicide, deprivation, and unemployment: record linkage study. BMJ 1998;317:1283-6. doi:10.1136/ bmj.317.7168.1283

38 Barak Y, Gale C. Suicide in long-term care facilities-the exception or the norm?JAMA Netw Open 2019;2:e195634. doi:10.1001/ jamanetworkopen.2019.5634
39 Mikkelsen ME, Still M, Anderson BJ, et al. Society of Critical Care Medicine's International Consensus Conference on

Prediction and Identification of Long-Term Impairments After Critical Illness. Crit Care Med 2020;48:1670-9. doi:10.1097/ CCM.0000000000004586

40 Jensen JF, Thomsen T, Overgaard D, Bestle MH, Christensen D, Egerod I. Impact of follow-up consultations for ICU survivors on post-ICU syndrome: a systematic review and meta-analysis. Intensive Care Med 2015;41:763-75. doi:10.1007/s00134-015-3689-1

41 Hatch R, Young D, Barber VS, Griffiths J, Harrison DA, Watkinson PJ. Anxiety, depression and post-traumatic stress disorder management after critical illness: a UK multi-centre prospective cohort study. Crit Care 2020;24:633. doi:10.1186/s13054-020-03354-y

Web appendix: Electronic supplement 